Original paper

\title{
Investigating centering, scan length, and arm position impact on radiation dose across 4 countries from 4 continents during pandemic: Mitigating key radioprotection issues
}

\author{
Shadi Ebrahimian a, Monica Oliveira Bernardo ${ }^{\mathrm{b}}$, Antônio Alberto Moscatelli ${ }^{\mathrm{b}}$, Juliana Tapajos ${ }^{\mathrm{c}}$, \\ Luciano Leitão Tapajós $^{c}$, Helen Jamil Khoury ${ }^{\mathrm{d}}$, Rosa Babaei ${ }^{\mathrm{e}}$, Hadi Karimi Mobin ${ }^{\mathrm{e}}$, \\ Iman Mohseni ${ }^{\mathrm{e}}$, Chiara Arru ${ }^{\mathrm{f}}$, Alessandro Carriero ${ }^{\mathrm{g}}$, Zeno Falaschi ${ }^{\mathrm{g}}$, Alessio Pasche ${ }^{\mathrm{g}}$, \\ Luca Saba $^{\mathrm{f}}$, Fatemeh Homayounieh ${ }^{\mathrm{a}}$, Bernardo C. Bizzo ${ }^{\mathrm{a}}$, Jenia Vassileva ${ }^{\mathrm{h}}$, \\ Mannudeep K. Kalra ${ }^{\text {a,* }}$
}

\footnotetext{
${ }^{a}$ Department of Radiology, Massachusetts General Hospital and the Harvard Medical School, Boston, MA, USA

${ }^{\mathrm{b}}$ Hospital Miguel Soeiro - UNIMED, Pontificia University Catholic of São Paulo - PUC-SP, Sorocaba, São Paulo, Brazil

${ }^{\mathrm{c}}$ Hospital Delphina Rinaldi Abdel Aziz, Manaus, Amazonas, Brazil

${ }^{\mathrm{d}}$ Nuclear Energy Department, Federal University of Pernambuco, Recife, Pernambuco, Brazil

e Department of Radiology, Firoozgar Hospital, Iran University of Medical Sciences, Tehran, Iran

${ }^{\mathrm{f}}$ Azienda Ospedaliera Universitaria di Cagliari, Cagliari, Italy

${ }^{g}$ Ospedale Maggiore della Carita', Novara, Italy

${ }^{\mathrm{h}}$ Radiation Protection of Patients Unit, International Atomic Energy Agency, Vienna, Austria
}

\section{A R T I C L E I N F O}

\section{Keywords:}

CT

Radiation dose

Patient mis-centering

Patient position

Radiation protection

\begin{abstract}
A B S T R A C T
Purpose: Optimization of CT scan practices can help achieve and maintain optimal radiation protection. The aim was to assess centering, scan length, and positioning of patients undergoing chest CT for suspected or known COVID-19 pneumonia and to investigate their effect on associated radiation doses.

Methods: With respective approvals from institutional review boards, we compiled CT imaging and radiation dose data from four hospitals belonging to four countries (Brazil, Iran, Italy, and USA) on 400 adult patients who underwent chest CT for suspected or known COVID-19 pneumonia between April 2020 and August 2020. We recorded patient demographics and volume CT dose index $\left(\mathrm{CTDI}_{\mathrm{vol}}\right)$ and dose length product (DLP). From thinsection CT images of each patient, we estimated the scan length and recorded the first and last vertebral bodies at the scan start and end locations. Patient mis-centering and arm position were recorded. Data were analyzed with analysis of variance (ANOVA).

Results: The extent and frequency of patient mis-centering did not differ across the four CT facilities $(>0.09)$. The frequency of patients scanned with arms by their side (11-40\% relative to those with arms up) had greater miscentering and higher $\mathrm{CTDI}_{\mathrm{vol}}$ and DLP at $2 / 4$ facilities $(\mathrm{p}=0.027-0.05)$. Despite lack of variations in effective

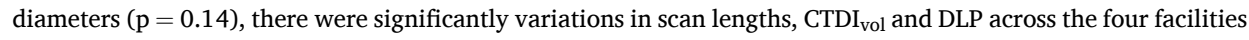
( $\mathrm{p}<0.001)$.

Conclusions: Mis-centering, over-scanning, and arms by the side are frequent issues with use of chest CT in COVID-19 pneumonia and are associated with higher radiation doses.
\end{abstract}

\section{Introduction}

Scan length, patient centering and positioning impact both image quality and radiation dose associated with CT scanning [1]. Scan length beyond the intended anatomy of interest may lead to unnecessary radiation dose and risks detection of incidental lesions which can require further testing and elevate patient anxiety [2]. Patient mis-centering interferes with adequate functioning of beam-shaping filters and lead

\footnotetext{
* Corresponding author: 75 Blossom Court, 02114 Boston, MA, USA.

E-mail address: mkalra@mgh.harvard.edu (M.K. Kalra).
} 
to under- or over-estimation of radiation dose across the cross-section of the body anatomy being imaged [3]. The latter is often associated with asymmetric and increased image noise and artifacts. Position of arms by side of the body during chest CT scanning is associated with artifacts and increased image noise despite an increase in radiation dose with automatic exposure-based modulation of applied tube current [4].

Most CT scanners and scan procedures demand close attention to patient positioning, centering and selection of scan length. Inappropriate patient positioning and centering can lead to increased image noise and artifacts that can compromise detection of incidental pathologies besides leading to greater than necessary radiation dose to the patients. These details represent non-trivial aspects of scanning that enable highest level of service and best hope for radiation dose optimization while retaining diagnostic quality of $\mathrm{CT}$, a modality that contributes to the largest fraction of radiation dose from medical imaging. However, demands and anxieties of a pandemic like the ongoing COVID19 infection can challenge or impede attention of CT technologist to these details over concern for personal safety and need to maintain minimum contact and maximum social distancing.

Imaging tools such as CT and radiography were and are being heavily used in the ongoing pandemic in patients with known or suspected infection, and that brings imaging personnel such as the CT technologists in close patient contact and increases their risk of contracting the virus. With adequate personal protective equipment (PPE) and precautions such risks can be reduced, but scarcity of such resources has been reported across the world [5]. Although prior studies have reported on variations on applications and radiation doses of CT in the ongoing COVID-19 crisis [6], to our best knowledge, there are no publications on how the practice of CT scanning has unfolded and how it has affected associated radiation doses. Thus, the purpose of our study was to assess centering, scan length, and positioning of patients undergoing chest CT for suspected or known COVID-19 pneumonia and to investigate their effect on associated radiation doses.

\section{Materials and Methods}

The institutional review boards of respective participating studies approved the study for retrospective evaluation of chest CT in patients with COVID-19 infection. All coauthors had access to de-identified study data. A study coauthor received research funding from Siemens Healthineers and Riverain Tech Inc for unrelated research. Other coauthors have no financial disclosures.

\section{Patients}

Our study included 400 patients from four CT facilities in one of these regions: South America (Hospital Miguel Soeiro - UNIMED, Sorocaba, São Paulo, Brazil; Delphina Rinaldi Abdel Aziz, Manaus, Amazonas, Brazil), Asia (Firoozgar Hospital, Iran University of Medical Sciences, Tehran, Iran), Europe (Ospedale Maggiore della Carita', Novara, Italy) and North America (Massachusetts General Hospital, Boston, Massachusetts, USA). Upon request from the participating facilities, their identity is blinded in the manuscript. All patients underwent a clinically-indicated chest CT for known or suspected COVID-19 pneumonia for assessing disease severity, treatment response or lack thereof, or complications.

Patients underwent chest CT at each facility - Facility A ( $n=98$ patients; mean age \pm standard deviation $58 \pm 14$ years; female: male 33 : 65), Facility B ( $\mathrm{n}=103$ patients; $60 \pm 17$ years; $36: 67)$, Facility C ( $\mathrm{n}=$ 99 patients; mean age \pm standard deviation $62 \pm 16$ years; female: male 33: 66), and Facility $\mathrm{D}(\mathrm{n}=100$ patients; mean age \pm standard deviation $76 \pm 11$ years; female: male 51: 49).

\section{Scanners and protocols}

All facilities performed their chest CT examinations with single-

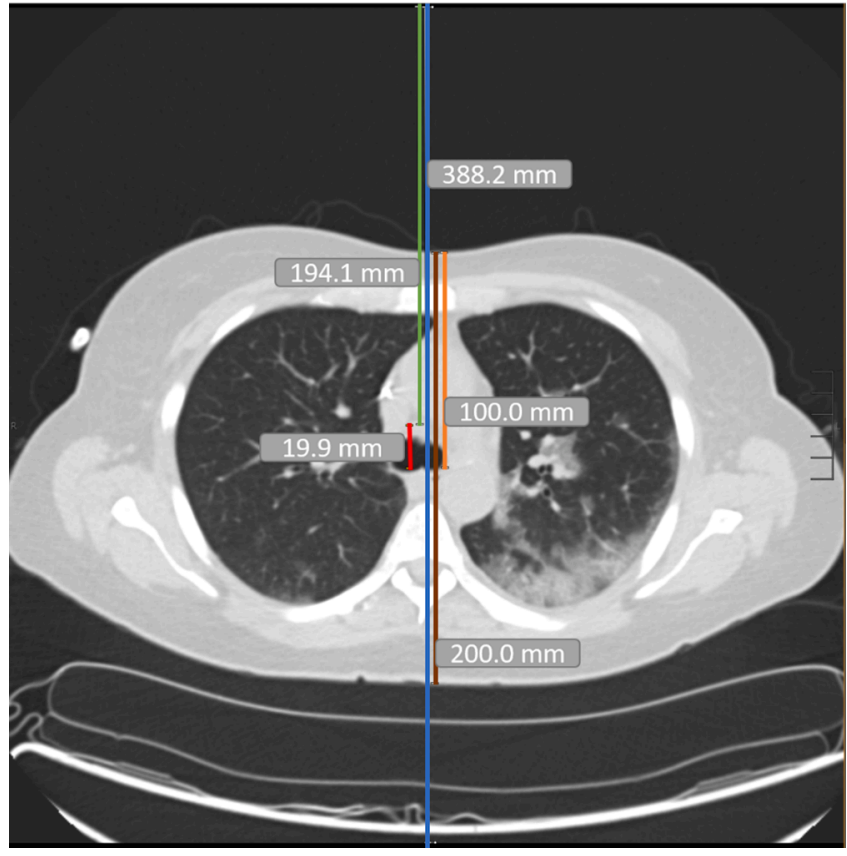

Fig. 1. Measurement of patient mis-centering on transverse CT images. The blue line represents the anteroposterior extent of the field of view and therefore it's half-length (green line) represents the isocenter of the field of view. The brown and orange lines (half length of the brown line) represent patient's anterior-posterior dimension and midpoint at the level of carina. The distance between the midpoints of the field of view and patient in red line represents mis-centering distance. (For interpretation of the references to colour in this figure legend, the reader is referred to the web version of this article.)

phase, non-intravenous contrast scan protocols. Only the index chest CT was included for each patient.

Facility A used either a 16-slice (GE Bright-Speed, GE Healthcare) or a 64-slice (Philips Brilliance, Philips Healthcare) multidetector-row CT scanners with the following scan parameters $-120 \mathrm{kV}$, vendor-specific automatic exposure control, 0.9:1 (Philips) or 1.375:1 pitch (GE), and $0.7-0.8 \mathrm{~s}$ gantry rotation time. CT image reconstruction factors included filtered back projection, soft tissue reconstruction kernels, and $1.25 \mathrm{~mm}$ section thickness.

At Facility B, all patients underwent chest CTs on a 16-slice, multidetector-row CT (Siemens SOMATOM Emotion 16, Siemens Healthineers) with $110-130 \mathrm{kV}, 30-50 \mathrm{mAs}$ (fixed tube current), 1.5:1 pitch, $16 \times 1.2 \mathrm{~mm}$ detector configuration, and 1-second gantry rotation time. Reconstruction parameters were filtered back projection technique, $2 \mathrm{~mm}$ section thickness and B20f (standard soft tissue) kernel.

Facility C patients received their single-phase imaging on a 128-slice multidetector-row CT (Philips Ingenuity Core, Philips Healthcare) at $120 \mathrm{kV}, 225 \mathrm{mAs}$ (automatic exposure control - Z-DOM), scannerselected pitch factor, 0.5-second gantry rotation time, and $64 \times 0.625$ $\mathrm{mm}$ detector configuration. Reconstruction parameters included filtered back projection, $1 \mathrm{~mm}$ section thickness, and Filter B (standard soft tissue reconstruction kernel).

Patients from Facility D received their CT on one of the four scanners (64-slice CT, GE Discovery 750HD; 64-96-slice CTs, Siemens Definition Force, Flash, or Edge). The scanning and reconstruction parameters included $100-120 \mathrm{kV}$, vendor-specific automatic exposure control, 0.9-1:1 pitch, 0.5-second gantry rotation time, 1-1.25 mm section thickness generated with iterative reconstruction technique (Admire, Siemens; ASIR, GE).

\section{Radiation dose descriptors}

Fully de-identified CT images of each patient were centrally collected 
Table 1

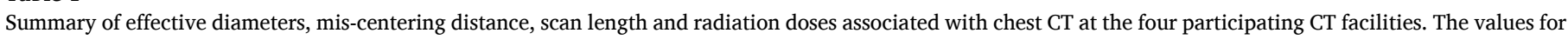

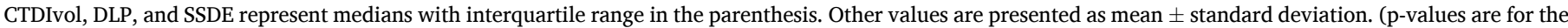

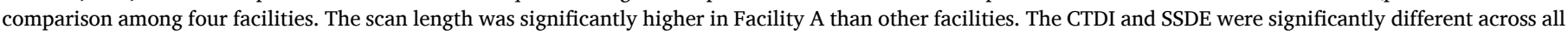
four facilities. DLP was not significantly different between facilities A and C, but were significantly higher than facilities B and D.) (R1-7).

\begin{tabular}{|c|c|c|c|c|c|}
\hline & Facility A F(A) & Facility B F(B) & Facility C F(C) & Acility D F(D) & p-value \\
\hline Effective diameter $(\mathrm{cm})$ & $27.7 \pm 3.6$ & $27.6 \pm 5.6$ & $28.5 \pm 3.1$ & $27.7 \pm 3.7$ & 0.141 \\
\hline Mis-centering distance (mm) & $16.9(8.0-30.2)$ & $16.6(8.8-31.0)$ & $13.6(7.1-24.5)$ & $21.1(10.6-29.4)$ & 0.205 \\
\hline Scan length (mm) & $400.0(304.1-400.0)$ & $317.9(391.4-338.0)$ & $311.0(295.0-330.0)$ & $300.0(279.7-329.3)$ & $<0.001$ [between $\mathrm{F}(\mathrm{A})$ and $\mathrm{F}(\mathrm{B}, \mathrm{C}, \mathrm{D})]$ \\
\hline $\mathrm{CTDI}_{\mathrm{vol}}$ (mGy) & $12(9-15)$ & $5(5-5)$ & $15(12-19)$ & $6(4.6-9)$ & $<\mathbf{0 . 0 0 1}[$ between $\mathrm{F}(\mathrm{A}, \mathrm{C})$ and $\mathrm{F}(\mathrm{B}, \mathrm{D})]$ \\
\hline DLP (mGy.cm) & 397 (302-477) & 179 (157-201) & $444(343-584)$ & $208(140-313)$ & $<\mathbf{0 . 0 0 1}$ [between all facilities] \\
\hline SSDE (mGy) & $16(11-19)$ & $7(7-8)$ & $19(15-22)$ & $8(6-12)$ & $<0.001$ [between $\mathrm{F}(\mathrm{A}, \mathrm{C})$ and $\mathrm{F}(\mathrm{B}, \mathrm{D})]$ \\
\hline
\end{tabular}

for analysis. A study coinvestigator (SE, with one-year post-doctoral research experience in radiology) recorded CT dose index volume $\left(\mathrm{CTDI}_{\mathrm{vol}}\right)$ and dose length product (DLP) from the dose information page or radiation dose structured reports (RDSR).

Since patient weights were not available from three of the four CT facilities, we recorded anteroposterior and lateral dimensions of each patient from their DICOM CT images at the level of tracheal carina. For chest CTs without skin to skin coverage, such measurements were recorded from the planning radiographs where available. Effective diameter was calculated as the square root of the product of anteroposterior and lateral dimensions [7]. We estimated size specific dose estimates by multiplying CTDI $_{\mathrm{vol}}$ with conversion factors derived from effective diameter lookup tables [8].

\section{Scan length, arm position, and centering}

Scan length was calculated from the scan start and end locations for each CT exam. In addition, we recorded the vertebral body number at the scan start and scan end locations since scan length can vary based on patients' height. Fig. 1 illustrates patient centering estimated at the level of the carina. First, we determined the center of the scan field of view in lung windows (scanner isocenter) and then patient center along a midline anterior-posterior line from patient's skin-to-skin. The distance between scanner isocenter and patient center represented the presence and extent of mis-centering.

\section{Statistical analysis}

Statistical analysis was performed in Microsoft EXCEL (Microsoft Inc., Redmond, Washington) using analysis of variance (ANOVA) to compare extent of mis-centering, scan length, arm position, patient size (effective diameter), CTDI ${ }_{\mathrm{vol}}$, DLP and SSDE. We used shapiro-wilk test to assess for normality of the data variables. Mean and standard deviations were estimated for quantitative variable. We performed Pearson correlation tests to find association between mis-centering, scan length, and CT radiation doses. We set $\mathrm{p}<0.05$ as the cut-off for statistical significance.

\section{Results}

Patients scanned at Facilities A, B, C had similar age and gender distribution ( $p>0.1$ ) whereas Facility $D$ had significantly older patients with more female than male patients $(\mathrm{p}<0.001)$. As noted in Table 1 , there was no difference in patient size (effective diameters) across the four participating facilities $(\mathrm{p}=0.141)$. However, there was a significant difference in radiation doses $\left(\mathrm{CTDI}_{\mathrm{vol}}\right.$, SSDE, and DLP) between the facilities $(\mathrm{p}<0.001)$. There was no significant correlation between miscentering distance with either effective diameters or with any of the radiation dose descriptors $\left(\mathrm{CTDI}_{\mathrm{vol}}\right.$, SSDE, DLP) $(\mathrm{r}<0.172, \mathrm{p}>0.083)$. There was a weak positive correlation between scan length and DLP $(r=$ $0.446, \mathrm{p}<0.001$ ) but no significant correlation was present between

Table 2

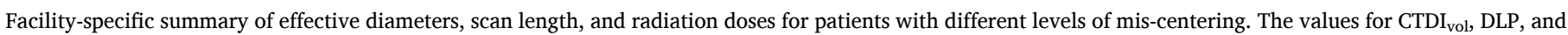
SSDE represent medians with interquartile range in the parenthesis. Other values are presented as mean \pm standard deviation.

\begin{tabular}{|c|c|c|c|c|c|}
\hline \multicolumn{2}{|c|}{ Mis-centering distance } & \multirow{2}{*}{$\begin{array}{l}\leq 0.5 \mathrm{~cm} \\
17 / 98(17.3 \%)\end{array}$} & \multirow{2}{*}{$\begin{array}{l}0.6-2.5 \mathrm{~cm} \\
45 / 98(45.9 \%)\end{array}$} & \multirow{2}{*}{$\begin{array}{l}\geq 2.6 \mathrm{~cm} \\
36 / 98(36.7 \%)\end{array}$} & \multirow{2}{*}{$\frac{\mathrm{p} \text {-value }}{-}$} \\
\hline Facility A & Number (\%) & & & & \\
\hline & Effective diameter $(\mathrm{cm})$ & $28.4 \pm 4.5$ & $28.1 \pm 3.1$ & $26.9 \pm 3.6$ & 0.255 \\
\hline & Scan length (mm) & $400.0(331.3-400.0)$ & $400.0(330.5-400.0)$ & $347.5(299.3-400.0)$ & 0.551 \\
\hline & $\mathrm{CTDI}_{\mathrm{vol}}(\mathrm{mGy})$ & $13(10-15)$ & $13(10-15)$ & $10(8-14)$ & 0.421 \\
\hline & DLP (mGy.cm) & $404(352-475)$ & $426(308-488)$ & $367(282-474)$ & 0.704 \\
\hline & SSDE (mGy) & $16(13-17)$ & $16(13-19)$ & $14(11-20)$ & 0.704 \\
\hline \multirow[t]{6}{*}{ Facility B } & Number (\%) & $17 / 103(16.5 \%)$ & $55 / 103(53.4 \%)$ & $31 / 103(30.1 \%)$ & - \\
\hline & Effective diameter $(\mathrm{cm})$ & $27.5 \pm 2.6$ & $28.1 \pm 2.5$ & $26.7 \pm 2.6$ & 0.060 \\
\hline & Scan length $(\mathrm{mm})$ & 331.9 (298.9-356.9) & $321.5(304.4-340.4)$ & $294.0(270.9-331.9)$ & 0.031 \\
\hline & $\mathrm{CTDI}_{\mathrm{vol}}(\mathrm{mGy})$ & $5(5-8)$ & $5(5-5)$ & $5(5-5)$ & 0.163 \\
\hline & DLP (mGy.cm) & $191(166-272)$ & $181(163-201)$ & $160(146-181)$ & 0.031 \\
\hline & SSDE (mGy) & $8(7-12)$ & $7(7-8)$ & $7(7-8)$ & 0.427 \\
\hline \multirow[t]{6}{*}{ Facility C } & Number (\%) & $15 / 99(15.2 \%)$ & $60 / 99(60.6 \%)$ & $24 / 99(24.2 \%)$ & - \\
\hline & Effective diameter $(\mathrm{cm})$ & $28.1 \pm 2.3$ & $28.7 \pm 3.2$ & $28.5 \pm 3.5$ & 0.825 \\
\hline & Scan length $(\mathrm{mm})$ & $309.0(295.0-327.0)$ & $314.0(292.0-327.8)$ & $303.0(293.0-331.8)$ & 0.101 \\
\hline & $\mathrm{CTDI}_{\mathrm{vol}}(\mathrm{mGy})$ & $13(10-17)$ & $15(11-19)$ & $16(13-19)$ & 0.145 \\
\hline & DLP (mGy.cm) & $396(297-549)$ & $438(340-590)$ & $506(396-582)$ & 0.516 \\
\hline & SSDE (mGy) & $16(14-22)$ & $19(15-22)$ & $21(17-24)$ & 0.145 \\
\hline \multirow[t]{6}{*}{ Facility D } & Number (\%) & $13 / 100(13 \%)$ & $51 / 100(51 \%)$ & $36 / 100(36 \%)$ & - \\
\hline & Effective diameter $(\mathrm{cm})$ & $27.2 \pm 2.8$ & $27.6 \pm 3.3$ & $28.2 \pm 4.4$ & 0.644 \\
\hline & Scan length (mm) & 277.5 (275.9-318.0) & $305.0(282.5-327.0)$ & $294.0(285.0-330.0)$ & 0.289 \\
\hline & $\mathrm{CTDI}_{\mathrm{vol}}(\mathrm{mGy})$ & $5(3-8)$ & $6(4-12)$ & $6(5-8)$ & 0.779 \\
\hline & DLP (mGy.cm) & $159(102-321)$ & $203(138-355)$ & $223(157-283)$ & 0.705 \\
\hline & SSDE (mGy) & $8(4-9)$ & $8(6-18)$ & $9(7-11)$ & 0.542 \\
\hline
\end{tabular}




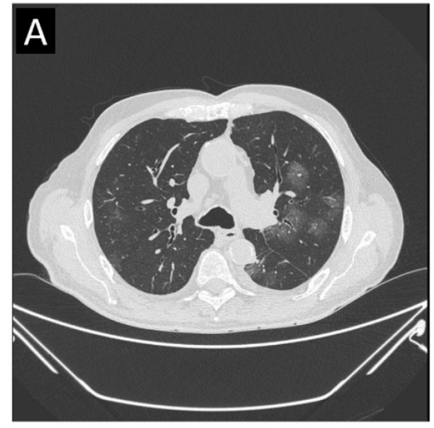

Effective diameter $=28 \mathrm{~cm}$ CTDIvol=10 $\mathrm{mGy}$ $\mathrm{DLP}=324 \mathrm{mGy} \cdot \mathrm{cm}$ Centering $=1.4 \mathrm{~mm}$
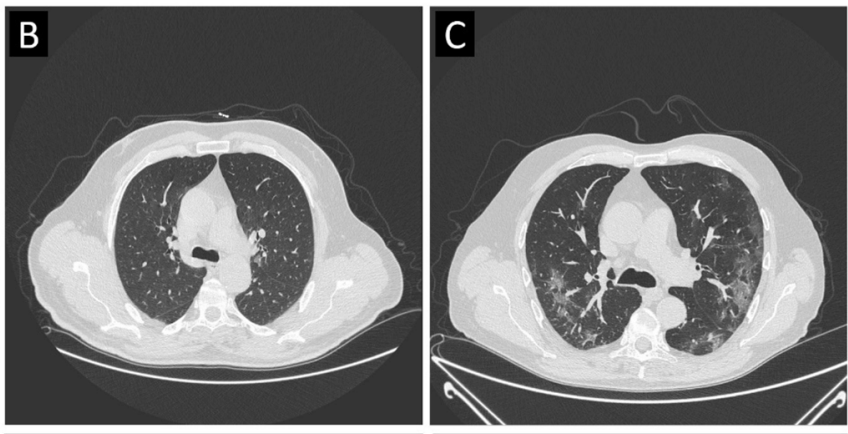

Effective diameter $=30$ CTDIvol $=18 \mathrm{mGy}$ $\mathrm{DLP}=741 \mathrm{mGy} . \mathrm{cm}$ Centering $=26 \mathrm{~mm}$
Effective diameter $=28$ CTDIvol=13 $\mathrm{mGy}$ DLP=384 mGy.cm Centering $=49 \mathrm{~mm}$

Fig. 2. The extent of mis-centering in three patients who underwent chest CT examinations for known COVID-19 pneumonia. Note presence of patchy multifocal groundglass opacities on patients A and C. There is a subtle groundglass nodular opacity in posterior aspect of the left upper lobe in patient B.

Table 3

Facility-specific summary of mis-centering distance, scan length, and CT radiation doses for patients with different effective diameters. The values for CTDI vol, $_{\text {, }}$ DLP, and SSDE represent medians with interquartile range in the parenthesis. Other values are presented as mean \pm standard deviation. The lack of significant statistical differences in CTDIvol, DLP and SSDE was related to use of fixed mAs at facility B. At facility D, a lack of significant difference in doses may be related to random variations in doses from use of data from four different CT scanners.

\begin{tabular}{|c|c|c|c|c|}
\hline \multicolumn{2}{|c|}{ Effective diameter } & \multirow{2}{*}{$\frac{\leq 30 \mathrm{~cm}}{73 / 98(74.5 \%)}$} & \multirow{2}{*}{$\frac{>30 \mathrm{~cm}}{25 / 98(25.5 \%)}$} & \multirow{2}{*}{$\frac{\text { p-value }}{-}$} \\
\hline Facility & Number (\%) & & & \\
\hline & Centering & $21.4 \pm 13.3$ & $14.3 \pm 13.6$ & 0.023 \\
\hline & Scan length & 400.0 & 400.0 & 0.595 \\
\hline & $(\mathrm{mm})$ & (299.0-400.0) & $(347.0-400.0)$ & \\
\hline & $\mathrm{CTDI}_{\mathrm{vol}}(\mathrm{mGy})$ & $10(8-13)$ & $15(13-15)$ & 0.001 \\
\hline & DLP (mGy.cm) & 351 (279-455) & $476(452-533)$ & $<0.001$ \\
\hline & SSDE (mGy) & $15(11-19)$ & $17(14-18)$ & 0.037 \\
\hline \multirow{7}{*}{$\begin{array}{c}\text { Facility } \\
\text { B }\end{array}$} & Number (\%) & $87 / 103(84.5 \%)$ & $16 / 103(15.5 \%)$ & - \\
\hline & Centering & $20.1 \pm 13.6$ & $14.4 \pm 8.0$ & 0.026 \\
\hline & Scan length & 314.4 & 321.5 & 0.101 \\
\hline & $(\mathrm{mm})$ & $(282.1-335.4)$ & $(305.4-344.7)$ & \\
\hline & $\mathrm{CTDI}_{\mathrm{vol}}(\mathrm{mGy})$ & $5(5-5)$ & $5(5-7)$ & 0.539 \\
\hline & DLP (mGy.cm) & 179 (156-199) & $178(163-241)$ & 0.966 \\
\hline & SSDE (mGy) & $7(7-8)$ & $7(6-8)$ & 0.033 \\
\hline \multirow{7}{*}{$\begin{array}{c}\text { Facility } \\
\text { C }\end{array}$} & Number (\%) & 72/99 (72.7\%) & $27 / 99(27.3 \%)$ & - \\
\hline & Centering & $17.4 \pm 13.5$ & $18.2 \pm 12.8$ & 0.809 \\
\hline & Scan length & 308.5 & 320.0 & 0.460 \\
\hline & $(\mathrm{mm})$ & (287.7-327.0) & (305.0-345.9) & \\
\hline & $\mathrm{CTDI}_{\mathrm{vol}}(\mathrm{mGy})$ & $14(10-17)$ & $20(17-20)$ & 0.001 \\
\hline & DLP (mGy.cm) & $405(321-515)$ & $590(519-631)$ & 0.001 \\
\hline & SSDE (mGy) & $19(15-22)$ & $21(18-23)$ & 0.011 \\
\hline \multirow{7}{*}{$\begin{array}{c}\text { Facility } \\
\text { D }\end{array}$} & Number (\%) & $77 / 100(77 \%)$ & $23 / 100(23 \%)$ & - \\
\hline & Centering & $22.5 \pm 16.5$ & $22.9 \pm 13.9$ & 0.919 \\
\hline & Scan length & 296.8 & 311.2 & 0.194 \\
\hline & $(\mathrm{mm})$ & $(282.2-325.7)$ & $(276.8-335.0)$ & \\
\hline & $\mathrm{CTDI}_{\mathrm{vol}}(\mathrm{mGy})$ & $6(4-9)$ & $6(5-11)$ & 0.591 \\
\hline & DLP (mGy.cm) & 205 (132-302) & $212(171-327)$ & 0.812 \\
\hline & SSDE (mGy) & $9(6-13)$ & $7(6-11)$ & 0.146 \\
\hline
\end{tabular}

scan length and effective diameter, CTDI $_{\mathrm{vol}}$ and SSDE ( $\left.\mathrm{p}>0.1\right)$.

\section{Centering}

Table 1 summarizes mis-centering distances at the four CT facilities.
Table 4

Facility-specific summary of effective diameters, and radiation doses for patients with different scan lengths of their chest CT. The values for CTDI ${ }_{\text {vol }}$, DLP, and SSDE represent medians with interquartile range in the parenthesis. Other values are presented as mean \pm standard deviation.

\begin{tabular}{|c|c|c|c|c|}
\hline \multicolumn{2}{|l|}{ Scan length } & \multirow{2}{*}{$\begin{array}{l}\leq 30 \mathrm{~cm} \\
24 / 98 \\
(24.5 \%)\end{array}$} & \multirow{2}{*}{$\begin{array}{l}>30 \mathrm{~cm} \\
74 / 98 \\
(75.5 \%)\end{array}$} & \multirow{2}{*}{$\frac{\text { p-value }}{-}$} \\
\hline Facility A & Number (\%) & & & \\
\hline & $\begin{array}{l}\text { Effective diameter } \\
(\mathrm{mm})\end{array}$ & $27.1 \pm 4.2$ & $27.9 \pm 3.3$ & 0.308 \\
\hline & $\mathrm{CTDI}_{\mathrm{vol}}(\mathrm{mGy})$ & $9(6-10)$ & $13(10-15)$ & $<0.001$ \\
\hline & DLP (mGy.cm) & 279 & 445 & $<0.001$ \\
\hline & SSDE (mGy) & $\begin{array}{l}(215-358) \\
11(10-13)\end{array}$ & $\begin{array}{l}(350-492) \\
17(14-19)\end{array}$ & $<0.001$ \\
\hline \multirow[t]{5}{*}{ Facility B } & Number (\%) & $35 / 103(34 \%)$ & 68/103 (66\%) & - \\
\hline & Effective diameter & $26.5 \pm 2.6$ & $28.1 \pm 2.4$ & 0.002 \\
\hline & $\mathrm{CTDI}_{\mathrm{vol}}(\mathrm{mGy})$ & $5(5-5)$ & $5(5-5)$ & 0.915 \\
\hline & DLP (mGy.cm) & $\begin{array}{l}155 \\
(139-165)\end{array}$ & $\begin{array}{l}181 \\
(172-205)\end{array}$ & $<0.001$ \\
\hline & SSDE (mGy) & 7 (7-9) & $7(7-8)$ & 0.487 \\
\hline \multirow[t]{6}{*}{ Facility C } & Number (\%) & $\begin{array}{l}37 / 99 \\
(37.4 \%)\end{array}$ & $\begin{array}{l}62 / 99 \\
(62.6 \%)\end{array}$ & - \\
\hline & Effective diameter & $27.8 \pm 2.6$ & $29.0 \pm 3.3$ & 0.037 \\
\hline & $\mathrm{CTDI}_{\mathrm{vol}}(\mathrm{mGy})$ & $15(11-18)$ & $16(12-19)$ & 0.336 \\
\hline & DLP (mGy.cm) & 394 & 517 & 0.002 \\
\hline & & (302-493) & (401-624) & \\
\hline & SSDE (mGy) & $19(16-23)$ & $20(15-22)$ & 0.336 \\
\hline \multirow[t]{5}{*}{ Facility D } & Number (\%) & $51 / 100(51 \%)$ & 49/100 (49\%) & - \\
\hline & Effective diameter & $27.3 \pm 3.9$ & $28.2 \pm 3.4$ & 0.210 \\
\hline & $\mathrm{CTDI}_{\mathrm{vol}}(\mathrm{mGy})$ & $6(4-8)$ & $7(5-9)$ & 0.192 \\
\hline & DLP (mGy.cm) & $\begin{array}{l}178 \\
(127-273)\end{array}$ & $\begin{array}{l}242 \\
(164-326)\end{array}$ & 0.009 \\
\hline & SSDE (mGy) & $7(6-11)$ & $9(6-13)$ & 0.312 \\
\hline
\end{tabular}

There was no significant difference in extent of mis-centering between the different facilities $(\mathrm{p}=0.205)$. When the extent of mis-centering was classified into three groups $(\leq 0.5 \mathrm{~cm}, 0.6-2.5 \mathrm{~cm}, \geq 2.6 \mathrm{~cm})$, less than one-fifth of the patients had precise centering in the CT gantry isocenter $(\leq 0.5 \mathrm{~cm})$; most patients were mis-centered at all CT facilities (Table 2). Patients within these three group of mis-centering had similar effective diameters $(\mathrm{p}>0.06)$ and radiation doses (both CTDI $_{\mathrm{vol}}$ and SSDE; $\mathrm{p}>$ 0.145) (Fig. 2). However, upon classification of patients based on their effective diameters $(<30 \mathrm{~cm}$ and $>30 \mathrm{~cm})$, larger patients had greater mis-centering than smaller patients in all sites and achieved statistical significance at $2 / 4$ sites $(\mathrm{p}=0.023-0.026)$ (Table 3 ). 

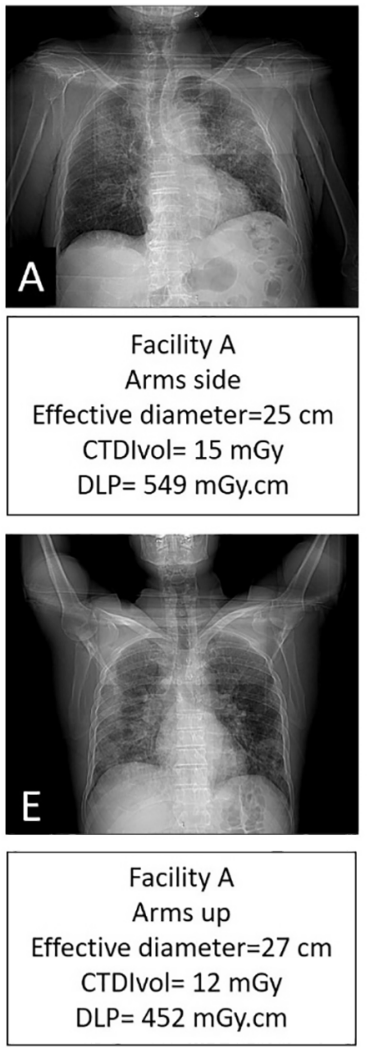
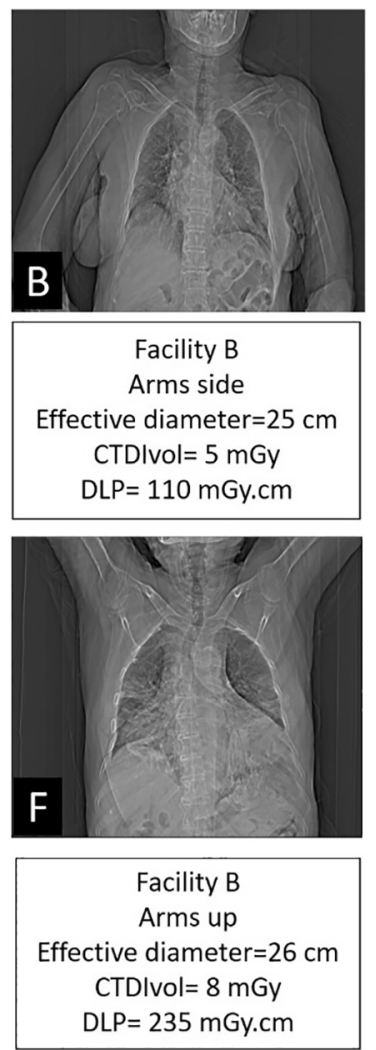
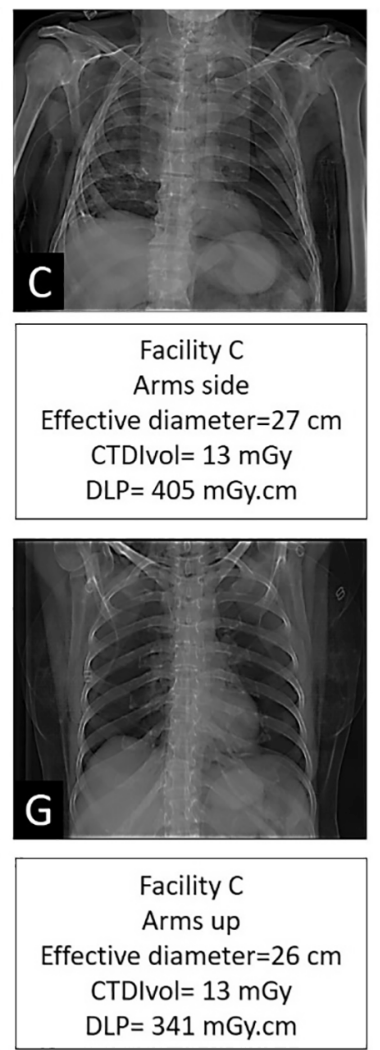
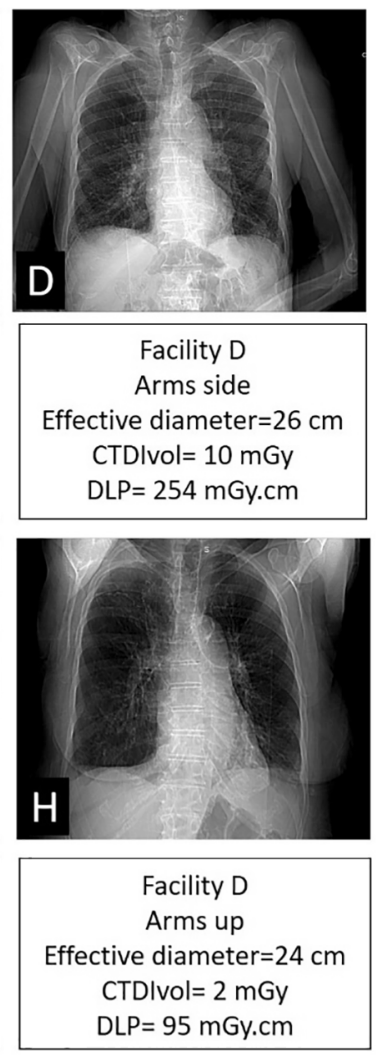

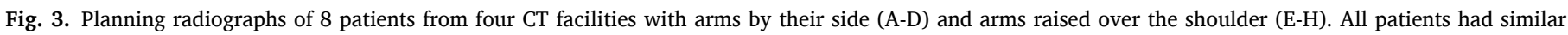
effective diameters.

Scan length

Table 2 shows that one of the four CT facilities had significantly longer scan lengths for chest CT as compared to other three facilities (p $<0.001$ ). However, the facility with the longest scan length did not have the highest CTDI $_{\text {vol }}$, DLP, or SSDE (Table 1). At 3/4 facilities, the scan length exceeded $30 \mathrm{~cm}$ for most chest CT examinations (Table 4). The effective diameters and associated DLP for patients from $2 / 3$ facilities with scan lengths $>30 \mathrm{~cm}$ were greater than those with $\leq 30 \mathrm{~cm}$. Table 3 describes the effect of effective diameter on scan lengths and associated radiation doses. At three of the $4 \mathrm{CT}$ facilities, chest CTs with greater mis-centering were not associated with longer scan length as compared to those with less mis-centering ( $p>0.194)$ (Table 2).

The most frequent scan start and end locations were $\mathrm{C} 7 / \mathrm{T} 1$ and T12/ L1 vertebral levels across all the four facilities. Although the vertebral level at the scan start location did not affect the scan length or the associated radiation doses ( $\mathrm{p}=0.927-0.050)$, scan end location at $\mathrm{L} 3$ or lower was associated with higher DLP (p 0.044 to $<0.001$ ).

\section{Arm position}

The facility-wise distribution of arm position during chest CT is summarized in Figs. 3 and 4. While patients from all facilities who were scanned with arms by their side had a greater mis-centering as compared to those scanned with arms above their shoulders, the difference did not achieve statistical significance ( $p>0.092)$. Conversely, patients from Facility A with arms by their side had significantly longer scan length as compared to those with raised arms $(\mathrm{p}<0.003)$. There was a small $(<10 \%)$ but significant difference in effective diameters of patients who underwent chest CT with different arm positions. The SSDE of patients scanned with arms by their side was greater than in those with raised arms in 3/4 CT facilities $(\mathrm{p}<0.041)$. The effect was most noticeable in
Facilities A and D, where despite a lower effective diameter, patients with arms by their side received a significantly higher radiation dose (both DLP and SSDE) as compared to those with arms raised over their shoulders.

\section{Discussions}

Our multicenter, international study found substantial variations in radiation doses associated with a single-phase, chest CT examinations performed in patients with COVID-19 pneumonia despite nearly identical distribution of patient age and cross-sectional effective diameters. We report frequent and greater mis-centering in most patients included in our study at all four participating facilities from Asia, Europe, North and South America. In comparison, prior studies in non-COVID-19 chest CT examinations reported mixed results. For example, Eberhard et al. [9], Akin-Akintayo et al. [10], and Habibzadeh et al. [11] reported mean mis-centering distances of $10.6,17$, and $16 \mathrm{~mm}$, respectively (compared to $17.6-22.6 \mathrm{~mm}$ in our study). Conversely, mis-centering in our study was lower than $24.9 \mathrm{~mm}$ reported by Li et al [12] and similar to $19 \mathrm{~mm}$ mis-centering distance in a study from Saltybaeva et al [13]. The extent of mis-centering in our study is likely related to lack of attention to centering, or lack of awareness on importance of good patient centering among CT radiographers/technologists at the participating sites. It is likely that such lack of attention was compounded by an intent to minimize patient contact in a setting of COVID-19 pandemic.

Although the effect of arm position on radiation dose associated with chest CT has been reported [14], its frequency and effect on scan length and mis-centering observed in our study has not been studied. We report that patients with arms by their side during chest CT are more likely to be mis-centered, scanned over longer area, and receive higher radiation dose. Although relationship between increased scan length and positioning of arms could have been coincidental, it is important to note that 


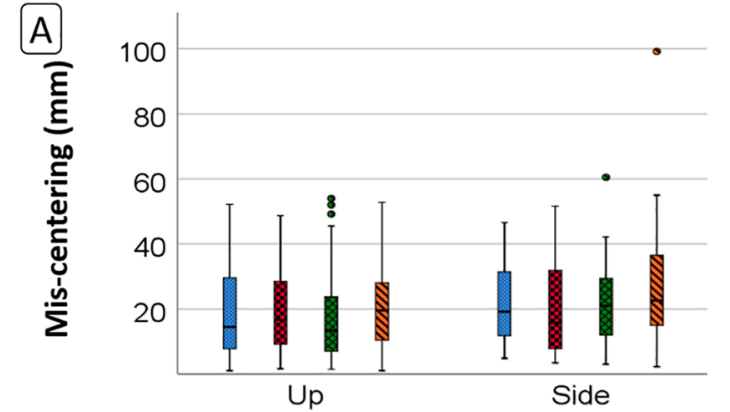

Arm position

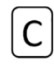

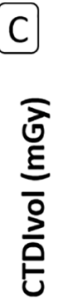

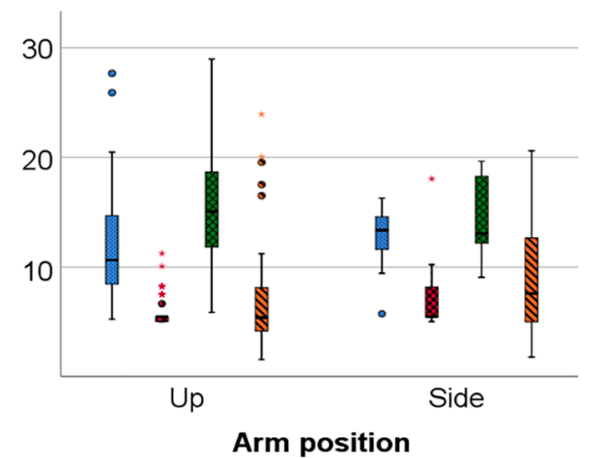

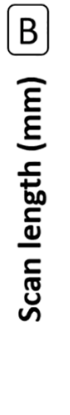

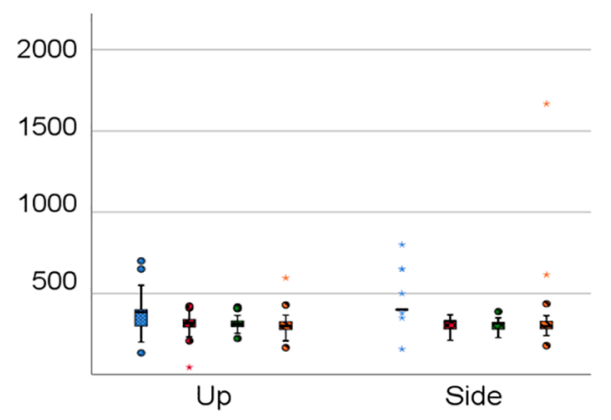

Arm position
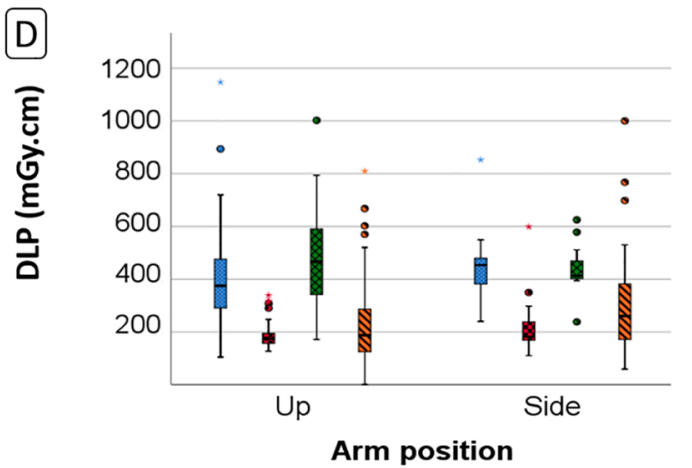

$\begin{array}{ll}\text { Facility C } & \text { Up }=88 ; \text { Side }=11 \\ \text { NFacility D } & \text { Up=60; Side }=40\end{array}$

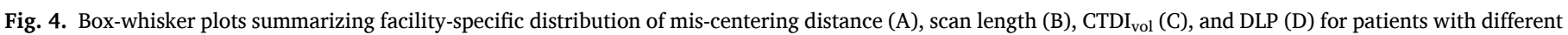

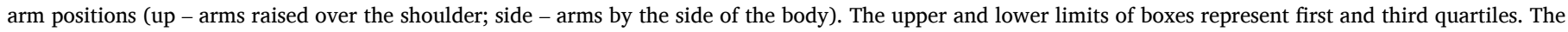
upper and lower limits of whiskers represent 9th and 91st percentiles. The dots represent the outliers.

when arms are positioned by the side of the body, they project on the chest in the lateral planning radiograph and can affect delineation of posterior costophrenic recesses and lower lungs which are difficult to see on the anteroposterior or posteroanterior planning radiographs. Relationship between mis-centering and arm positioning may be coincidental or related to the fact that positioning of the arms by the side of the patient impairs technologists' ability to judge the center of patient's chest. Although we could not specifically assess the reason for chest CT with arms by the side of the body, it is likely that the higher frequency of arms by the side resulted from a combination of shoulder pain/ arthropathy, presence of multiple lines and tubes in sick, ventilated patients, as well as from an attempt to minimize patient contact [15]. Likewise, longer than necessary scan lengths, notably extending below L2 or L3 vertebral body level increased the associated DLP.

A weak correlation between the scan length and DLP $(r=0.446)$ was likely related to the fact that most participating sites (3/4) used automatic tube current modulation which adapts tube current to patient size. As patient size (measured with effective diameter) was not correlated with the scan length, the tube current and therefore the applied radiation dose (CTDIvol at fixed tube potential) varied independently of the scan length and led to a weak correlation with scan length.

The chief implication of our study is the extent of variations in patient centering and scan lengths in patients undergoing chest CT for suspected or known COVID-19 infection. Such efforts can help of being better image quality with less artifact and aid in radiation dose optimization regardless of the clinical indication for scanning (with or without COVID-19 infection). It is imperative that imaging departments and hospital provide high quality PPE to CT radiographers/technologists so that they can serve with full confidence as the frontline workers involved in treating patients with contagious or communicable diseases.
When available, technologists should change the table height from the console room without entering the scan room if they notice miscentering on the planning radiographs. Our study also calls for enhanced effort from the CT industry to bring automatic centering and patient positioning techniques into real-world scanners regardless of their make, model, and year of installation. Two main CT vendors have reported on capabilities of automatic patient positioning software and hardware options to reduce mis-centering and select appropriate scan length. Li et al [12] reported on use of planning radiograph-based automatic patient re-centering technique in 2007 (GE) although unfortunately, their technique was never commercialized. More recent publications describe a commercially available, artificial intelligence-based technique (Siemens) for automatic patient centering and scan length determination on some of its scanners [13]. While these vendors deserve kudos for their efforts to identify with the issues and come up with excellent solutions, more is needed from them at the time of the current and future pandemics in an era of globalization and shrinking distances, so that we can maintain better CT image quality, reduce radiation dose, and make scanning safer for our CT technologists without requiring them to pay extra attention to details (centering and scan length prescription) where automated techniques have a proven track record of success $[12,13]$. CT vendors can also help reduce mis-centering and over-scanning by displaying their extent as well as their effects on image quality (such as increase in noise or artifacts) and radiation dose to the patients undergoing CT. On our part, we have conveyed findings of our study to the respective facilities so that CT technologists can pay more attention to patient positioning, centering and prescription of optimum scan length.

There are several limitations in our study. First, our study was limited to one or two CT facilities per country and a small number of 
patients and may not reflect a universal or common practice. Since, all participating CT facilities represented large, referral hospitals, we cannot comment on frequency and effect of mis-centering, arm position, and scan length on smaller CT facilities. Second, radiation doses across different CT facilities cannot be compared across different sites due to substantial differences in scanner technologies in these facilities. Third, we did not assess the effect of arm position or mis-centering on diagnostic quality of the exam since those effects have been documented in prior studies [14]. Fourth, we did not have information on patient location at the time of scanning from all facilities. Thus, we cannot establish if hospitalized or critical care unit patients with advanced or complicated disease have higher frequency of mis-centering, arms by the side of their body, and longer than necessary scan lengths. Fifth, manual measurement of mis-centering can lead to errors despite our attempt to double-check each measurement. We did not have access to any automated software or method of estimating mis-centering from CT images. Sixth, we did not have access to patients' weight at the time of scanning from 3/4 CT facilities. Instead, we used effective diameter as a surrogate for patient size which is a better marker of cross-sectional size than body weight. However, a lack of significant differences in radiation doses (summarized in table 3) at some facilities for patients with different effective diameters may have been related to inadequate sample size or arbitrary classification of effective diameter $(\leq 30$ and $>30 \mathrm{~cm})$. Seventh, none of the participating facilities recorded their patients' height; this complicates comparison of scan length and DLP. However, information on vertebral body levels at scan start and end locations helped us compare scan lengths and DLP across different facilities. Finally, due to retrospective nature of the study, we could not capture the information on change in scan parameters and radiation doses as a result of patient mis-centering.

\section{Conclusion}

In conclusion, mis-centering, over-scanning, and arms by the side are frequent in patients with COVID-19 pneumonia at all four participating international CT facilities. These issues increase associated radiation dose and result in lower image quality. Imaging departments and CT technologists must pay close attention to patient positioning and avoid over-scanning patients while scanning them, when possible, with their arms raised above the shoulders.

\section{References}

[1] Kaasalainen T, Palmu K, Reijonen V, Kortesniemi M. Effect of patient centering on patient dose and image noise in chest CT. AJR Am J Roentgenol 2014;203(1): 123-30. https://doi.org/10.2214/ajr.13.12028.

[2] Atalay MK, Walle NL, Egglin TK. Prevalence and nature of excluded findings at reduced scan length CT angiography for pulmonary embolism. J Cardiovasc Comput Tomogr 2011;5(5):325-32. https://doi.org/10.1016/j.jcct.2011.08.001.

[3] Ali Khawaja RD, Singh S, Padole A, Otrakji A, Lira D, Zhang D, et al. Point organ radiation dose in abdominal CT: effect of patient off-centering in an experimental human cadaver study. Radiat Protect Dosimetry 2017;175(4):440-9. https://doi. org/10.1093/rpd/ncw371.

[4] Kahn J, Grupp U, Maurer M. How does arm positioning of polytraumatized patients in the initial computed tomography (CT) affect image quality and diagnostic accuracy? Eur J Radiol 2014;83(1):e67-71. https://doi.org/10.1016/j. ejrad.2013.10.002.

[5] Cohen J, Rodgers Y. Contributing factors to personal protective equipment shortages during the COVID-19 pandemic. Prev Med 2020;141:106263. https:// doi.org/10.1016/j.ypmed.2020.106263.

[6] Homayounieh F, Holmberg O, Al Umairi R, Aly S, Basevičius A, Costa PR, et al. Variations in CT utilization, protocols, and radiation doses in COVID-19 pneumonia: results from 28 countries in the IAEA Study. Radiology 2020;10: 203453. https://doi.org/10.1148/radiol.2020203453.

[7] https://www.aapm.org/pubs/reports/RPT_220.pdf, [accessed 30 January 2021].

[8] https://www.aapm.org/pubs/reports/RPT_204.pdf, [accessed 30 January 2021].

[9] Eberhard M, Blüthgen C, Barth BK, Frauenfelder T, Saltybaeva N, Martini K. Vertical off-centering in reduced dose chest-CT: impact on effective dose and image noise values. Acad Radiol 2020;27(4):508-17. https://doi.org/10.1016/j. acra.2019.07.004.

[10] Akin-Akintayo OO, Alexander LF, Neill R, Krupinksi EA, Tang X, Mittal PK, et al. Prevalence and severity of off-centering during diagnostic CT: observations from 57,621 CT scans of the chest, abdomen, and/or pelvis. Curr Probl Diagn Radiol 2019;48(3):229-34. https://doi.org/10.1067/j.cpradiol.2018.02.007.

[11] Habibzadeh MA, Ay MR, Asl AR, Ghadiri H, Zaidi H. Impact of miscentering on patient dose and image noise in x-ray CT imaging: phantom and clinical studies. Phys Med 2012;28(3):191-9. https://doi.org/10.1016/j.ejmp.2011.06.002.

[12] Li J, Udayasankar UK, Toth TL, Seamans J, Small WC, Kalra MK. Automatic patient centering for MDCT: effect on radiation dose. AJR Am J Roentgenol 2007;188(2): 547-52. https://doi.org/10.2214/ajr.06.0370.

[13] Saltybaeva N, Schmidt B, Wimmer A, Flohr T, Alkadhi H. Precise and automatic patient positioning in computed tomography: avatar modeling of the patient surface using a 3-dimensional camera. Invest Radiol 2018;53(11):641-6. https:// doi.org/10.1097/rli.0000000000000482.

[14] Brink M, de Lange F, Oostveen LJ, Dekker HM, Kool DR, Deunk J, et al. Arm raising at exposure-controlled multidetector trauma CT of thoracoabdominal region: higher image quality, lower radiation dose. Radiology 2008;249(2):661-70. https://doi.org/10.1148/radiol.2492080169.

[15] Kalra MK, Homayounieh F, Arru C, Holmberg O, Vassileva J. Chest CT practice and protocols for COVID-19 from radiation dose management perspective. Eur Radiol 2020;30(12):6554-60. https://doi.org/10.1007/s00330-020-07034-x. 